\title{
Probing Glass Transition of Ultrathin Polymer Films at a Time Scale of Seconds Using Fast Differential Scanning Calorimetry
}

\author{
Mikhail Yu. Efremov, Eric A. Olson, Ming Zhang, Zishu Zhang, and \\ Leslie H. Allen*
}

Department of Materials Science and Engineering and Coordinated Science Laboratory, University of Illinois at Urbana-Champaign, Urbana, Illinois 61801

Received December 15, 2003; Revised Manuscript Received April 9, 2004

\begin{abstract}
UItrasensitive, thin-film, differential scanning calorimetry is used to determine the glasstransition temperature of 3-400 nm thick, spin-cast films of polystyrene, poly (2-vinyl pyridine), and poly (methyl methacrylate) on a platinum surface. The technique used here is modified to characterize the glass transition over a time scale of seconds rather than milliseconds. No appreciable dependence of the glass-transition temperature on thickness is observed over the entire range used. The results are discussed in comparison with previous calorimetric measurements, characterized by the glass-transition time scale of milliseconds.
\end{abstract}

\section{Introduction}

Gains made in manipulating and characterizing material on the microscale contribute significantly to modern science and industry, including microel ectronics, medicine, and biology. Examination of glass transition in polymer films under $100 \mathrm{~nm}$ thick, particularly concerning dependence of glass-transition temperature $\mathrm{T}_{\mathrm{g}}$ on film thickness, has attracted extensive attention. ${ }^{1-4}$ A vast variety of experimental methods-ellipsometry, Brillouin light scattering, X-ray reflectivity, positron lifetime spectroscopy, dielectric relaxation, local thermal analysis, AFM, etc.-have been utilized. However, the standard technique for glass-transition research in bulk materials-differential scanning calorimetry (DSC)-was not available for ultrathin polymer films until our recent advances in thin-film DSC (TDSC or "nanocalorimetry")..$^{5}$ The importance of cal orimetric measurements lies in the fact that it can measure fundamental thermodynamic properties such as heat capacity.

In our recent publication of nanocalorimetric investigations of polystyrene, poly (2-vinyl pyridine), and poly (methyl methacrylate) spin-cast films on a platinum surface, no appreciable dependence of glass-transition temperature over the thickness range from hundreds of nanometers down to $3 \mathrm{~nm}$ was observed. ${ }^{6}$ However, several other techniques, primarily ellipsometry, yield data which point to significant thickness dependence of $\mathrm{T}_{\mathrm{g}} \cdot{ }^{1-4}$ The magnitude of the change of $\mathrm{T}_{\mathrm{g}}$ with thickness in those experiments depends mostly on the type of polymer and substrate and typically achieves an increase or decrease of several tens of degrees of $\mathrm{T}_{\mathrm{g}}$ from bulk samples down to about $10 \mathrm{~nm}$ thick films. One of our suppositions, which would make our calorimetric data consistent with results obtained by other methods, is that the absence of size dependence of $T_{g}$ in nanocalorimetric experiments may be related to the high heating and cooling rates. ${ }^{6}$ These rates are $20-200 \mathrm{~K} / \mathrm{ms}$ for heating and about $1-2 \mathrm{~K} / \mathrm{ms}$ for cooling in the glass-transition region of the investigated polymers $\left(100-200{ }^{\circ} \mathrm{C}\right)$. Nanocalorimetric data are

* To whom correspondence should be addressed. E-mail: L-ALLEN9@iuc.edu.

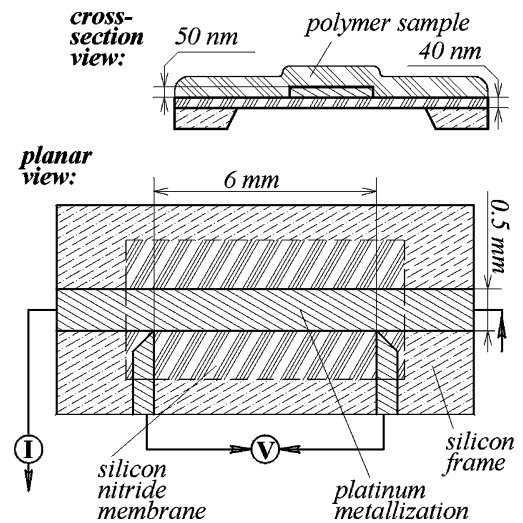

Figure 1. Cross-section and planar views of a typical TDSC sensor with a polymer sample on it (not to scale).

acquired upon heating. These high rates are inherent in the TDSC technique. In contrast, most other techniques take measurements on time scales on the order of seconds and minutes, not milliseconds.

To check this hypothesis, we make a special modification of the nanocal orimetry technique, which was briefly described elsewhere. ${ }^{5}$ The idea is to probe thermophysical properties (by nanocalorimetry) of polymer films as a function of annealing temperature. Annealing the sample immediately before the calorimetric scans can be made at time scales compatible with those of ellipsometric and other low scanning-speed techniques. In this case, the data from nanocal orimetric scans are used only as an indicator of changes in the sample when the annealing temperature reaches the glass-transition region. A similar approach was used, for example, in the study of glass transition on the surface of poly(vinyl alcohol) by sum-frequency vibrational spectroscopy. ${ }^{7}$

\section{Experimental Section}

2.1. Nanocalorimetry Technique. The nanocal orimetric technique is discussed in detail elsewhere. ${ }^{8-10}$ Briefly, our nanocalorimetric sensor (Figure 1) consists of a thin, freestanding, silicon nitride membrane $(40-400 \mathrm{~nm}$ thick and several millimeters in height and width) supported by a silicon frame. Sensors with thick membranes are used for thick (70$80 \mathrm{~nm}$ and above) polymer films in order to resist the internal 


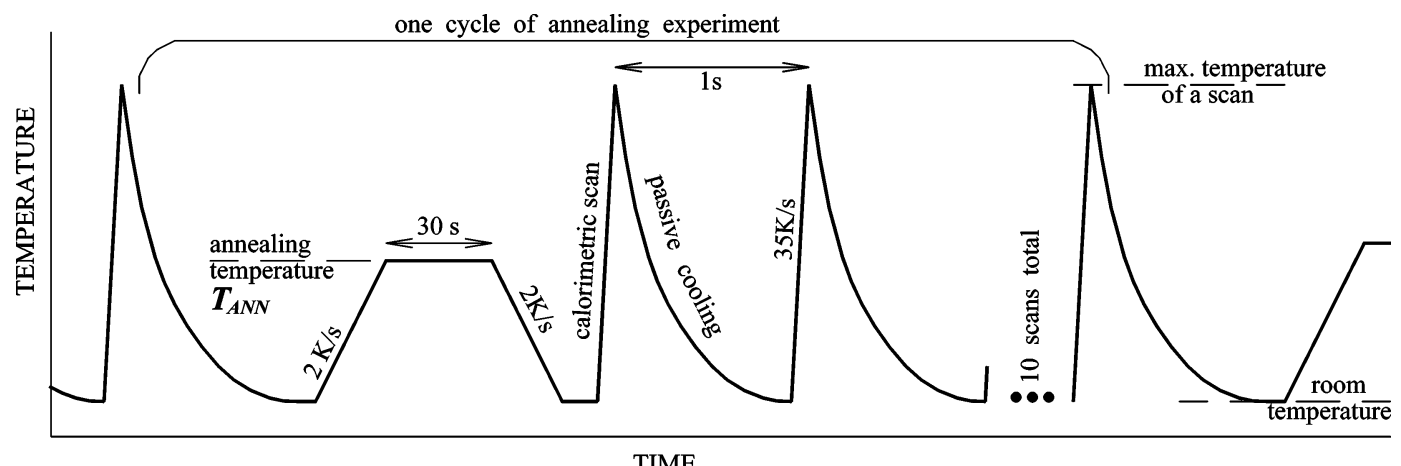

Figure 2. Structure of the annealing experiment.

stress in silicon nitride/polymer system. A platinum heater strip ( $\sim 50 \mathrm{~nm}$ thick) is deposited onto one side of the membrane. By inducing a current through it, such a heater serves both to heat the membrane and to simultaneously measure its temperature. The heater's resistance is calibrated against temperature prior to the experiments. Very fast heating, varied from 20 to $200 \mathrm{~K} / \mathrm{ms}$, allows the sensor to operate in nearly adiabatic conditions. The calorimetric cell, consisting of thin membrane and heater strip only, has exceptionally low addenda, which is a key attribute of the high sensitivity of this method.

In the differential scheme, one sensor, used as is, serves as the reference one; the sample is placed on another sensor. During the experiment, a current pulse passes through the heaters of the sample and reference simultaneously. Differential voltage $\Delta \mathrm{V}$ between the sample and reference sensors along with the voltage and current across the heaters are measured and used for power and temperature calculations and, finally, for calculation of $C_{p}(T)$, also known as the cal orimetric curve.

2.2. Heat Capacity Analysis. Methods for heat capacity calculation using voltage and current experimental data are discussed in detail in ref 9 . To obtain general calorimetric data for the samples in this investigation, the more accurate "main" method with three corrections ${ }^{9}$ is used. This method is useful, for example, to measure the amount of the polymer on the sensor (in the calorimetric cell). However, for processing of calorimetric scans in the annealing experiments, the simplified method is used. As emphasized in ref 9 , the derivative of the differential voltage $\Delta \mathrm{V}$ over time, $\mathrm{d} \Delta \mathrm{V} / \mathrm{dt}$, contains the major part of the information about thermal processes in the sample. Heat capacity of the sample as a function of temperature $C_{P}(T)$ can be well approximated by the linear transformation of the $\mathrm{d} \Delta \mathrm{V} / \mathrm{dt}(\mathrm{T})$ dependence, and all three corrections add relatively smooth terms (in comparison with $\mathrm{d} \Delta \mathrm{V} / \mathrm{dt}(\mathrm{T})$ function) to the $C_{P}(T)$ dependence. This means that if the purpose of the measurement is to trace changes in the thermal properties of the sample caused by changes in the sample treatment (but not to quantify the properties), the $\mathrm{d} \Delta \mathrm{V} / \mathrm{dt}(\mathrm{T})$ function will be as useful as the accurate $C_{P}(T)$ dependence. Moreover, in this case of using TDSC technique only as a probe of changes caused by different annealing procedures, the $\mathrm{d} \Delta \mathrm{V} / \mathrm{dt}(\mathrm{T})$ function as a data output has advantages over $C_{P}(T)$. The accuracy of the "main" method of $C_{P}(T)$ calculations with the corrections comes at a price of complicated data treatment, which introduces additional spurious signals in the output. While the overall accuracy of the result is enhanced, the quality of separation between outputs for the measurements under close, but not identical, conditions, can be deteriorated. Additionally, the simplicity and quick turnaround of $\mathrm{d} \Delta \mathrm{V} / \mathrm{dt}(\mathrm{T})$ calculation is essential in the measurements presented here, where typically 400 dependencies are analyzed and compared for each film sample.

2.3. Sample Preparation. Three types of monodispersed atactic polymer were chosen for this work: polystyrene, $M_{w}=120200, M_{w} / M_{n}=1.04$ (PS), poly(2-vinyl pyridine), $\mathrm{M}_{\mathrm{w}}=138000, \mathrm{M}_{\mathrm{w}} / \mathrm{M}_{\mathrm{n}}=1.30(\mathrm{PVP})$, and poly(methyl methacrylate), $M_{w}=105400, M_{w} / M_{n}=1.04$ (PMMA). To observe effects of different molecular weight, several experiments with high molecular weight polystyrene, $M_{w}=10200000$, $M_{w} / M_{n}=1.22(P S-H)$, were performed. All samples were obtained from Polymer Source, Inc. These glass-forming polymers are widely used for studies of glass transition in thin films ${ }^{4}$ and were used in our previous direct nanocal orimetric studies. ${ }^{6}$ Annealing experiments were part of nanocal orimetric experiments discussed in ref 6 , so the sample preparation and testing procedure are described there. In short, the films of polymers were formed by the spin-cast method from polymer solutions on the platinum heater of the calorimetric sensor. Film thickness was controlled both by varying the concentration of solution (0.06-7.5\%) and the speed of rotation (300010000 rpm, typically $10000 \mathrm{rpm}$ ). Solvent used for PS and PMMA was toluene, and for PVP it was n-butanol. The average thickness of film on the heater was calculated from the room temperature heat capacity value, using the specific heat capacity of the bulk substance.

Additionally, the local film thicknesses on the heater at 15 points were measured (both before and after calorimetric measurement) ex situ by focused ellipsometry (Focus Ellipsometer FE-III, Rudol ph Technologies, Inc) with a spot size of $12 \times 24 \mu \mathrm{m}$. The deviation of any local thickness from the average value does not exceed $\pm 10 \%$ except for the thickest $(56 \mathrm{~nm}) \mathrm{PS}-\mathrm{H}$ sample. The agreement between both calorimetric and ellipsometric types of thickness measurements in $90 \%$ cases is better than $\pm 10 \% \pm 0.2 \mathrm{~nm}$. The roughness of selected samples of PS, PVP, and PMMA on sensors was measured by atomic force microscopy (Digital Instruments Dimension 3000) after all calorimetric and ellipsometric measurements. The roughness of the thinnest films $(1-2 \mathrm{~nm})$ on the heater is equal to the roughness of the bare heater itself-about $1.5 \mathrm{~nm}$ of rms value. The roughness of thicker films decreases gradually with thickness. The AFM measurements also confirm that all polymers wet the surface of the platinum heater. After spin casting the polymer and taking ellipsometry measurements, the samples were preannealed in a vacuum for $1000 \mathrm{~s}$ at $140^{\circ} \mathrm{C}$ for PS and PVP and at $160{ }^{\circ} \mathrm{C}$ for PMMA to maintain reproducibility of the thermal properties of the films throughout the whole experiment. Experiments were performed under high vacuum (1-4 × 10-8 Torr).

2.4. Experimental Procedure. The procedure of annealing measurement is illustrated in Figure 2. After the end of a previous cycle, the sample was heated $2 \mathrm{~K} / \mathrm{s}$ up to the specific annealing temperature, $\mathrm{T}_{\mathrm{ANN}}$, isothermally annealed at this temperature for $30 \mathrm{~s}$, and then cooled to room temperature at $2 \mathrm{~K} / \mathrm{s}$. Within a few seconds after the cooling period, 10 consecutive nanocalorimetry scans were performed at $1 \mathrm{~s}$ intervals. The scans were made starting from room temperature up to $240^{\circ} \mathrm{C}$ for PS and PVP and up to $260^{\circ} \mathrm{C}$ for PMMA, with heating rates of $30-40 \mathrm{~K} / \mathrm{ms}$. After reaching the maximum temperature during each scan, the sample was passively cool ed at a rate of $1-2 \mathrm{~K} / \mathrm{ms}$ over the $200-100{ }^{\circ} \mathrm{C}$ range (where the glass transition occurs). After the last scan, the annealing measurement is repeated at different $T_{\text {ANN }}$.

The lower limit of $\mathrm{T}_{\text {ANN }}$ was $30{ }^{\circ} \mathrm{C}$, and the upper limit of $\mathrm{T}_{\text {ANN }}$ is $140{ }^{\circ} \mathrm{C}$ for PS and PVP and $200^{\circ} \mathrm{C}$ for PMMA. During the experiment, $\mathrm{T}_{\text {ANN }}$ was changed monotonically by fixed 


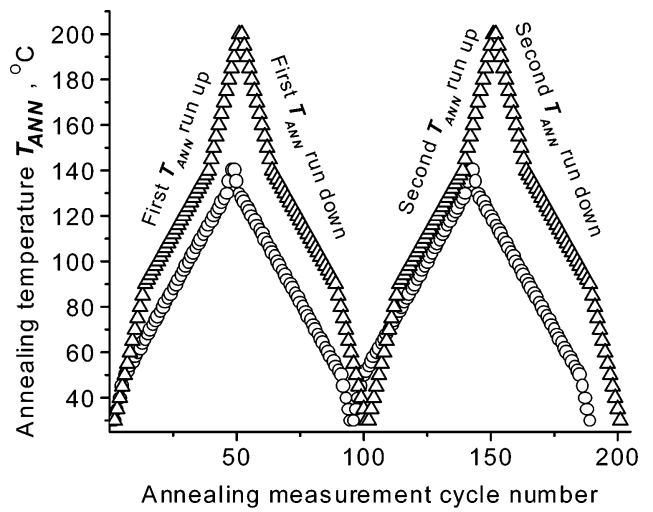

Figure 3. Schedule of $T_{\text {ANN }}$ change during the annealing experiment: $(\triangle)$ PMMA, (O) PS and PVP.

increments/decrements from the lower limit to the upper limit ( $T_{\text {ANN }}$ run up) and back to the lower limit ( $T_{\text {ANN }}$ run down). Such large cycles were repeated twice. I ncrements of $\mathrm{T}_{\text {ANN }}$ were $5 \mathrm{~K}$ far away from glass-transition region and $2 \mathrm{~K}$ close to it. The schedule of $\mathrm{T}_{\text {ANN }}$ changes during the experiment is illustrated in Figure 3.

The calorimetric data are sensitive to tiny differences in the thermal history. For example, the data from the first scan after annealing is sensitive to the time interval between the end of the annealing procedure and the start of the scan. Because of this, the entire sequence of annealing cycles of a measurement (typically about 200 cycles of annealing-scanning) was done in a fully automatic regime to ensure adequate reproduci bility of the thermal treatment of the sample.

2.5. Temperature Nonuniformity Over the Calorimetric Cell during the Annealing Procedure. The temperature regulation for the annealing procedure was performed by changing the current through the metal heater strip of the sensor. While the heating speed for a calorimetric scan is high enough to ensure practically adiabatic conditions and good uniformity of the temperature distribution within the calorimetric cell, slow heating during the annealing procedure is far from adiabatic and the temperature distribution within the calorimetric cell is significantly broader. The obvious consequence of the temperature nonuniformity during the annealing procedure is the broadening of the glass-transition region and, therefore, more uncertainty in the $\mathrm{T}_{\mathrm{g}}$ assignment.

To estimate the nonuniformity of the temperature distribution over the calorimetric cell during the isothermal part of annealing, finite element modeling of the isothermal regime was performed. The modeling software was made on the basis of the Partial Differential Equation Toolbox of MATLAB software (release 12). ${ }^{11}$ The model consists of the system of two equations-the Laplace elliptic equation for el ectrostatics of conductive media problem and the elliptic version of the heat balance equation for the steady-state conditions. Each equation was discretized by the finite element method, using meshes with more than $10^{5}$ triangles and an adaptive mesh refinement algorithm.

Predefined parameters of the model are the sensors' dimensions, the experimentally measured el ectrical conductivity of the heater, the experimental temperature coefficient of metallization resistance, and the lateral heat conductivity of the platinum metallization of the sensors, estimated using the measured electrical conductivity of the metallization and the Wiedemann-Franz law. Heat conductivity of the polymer was neglected. The variable model parameters are the lateral heat conductivity of the silicon nitride membrane and the emissivity of the metallization. Emissivity of the membrane and polymer is assumed to be zero. It is also supposed that all heat conductivity and emissivity parameters do not depend on temperature. The variable parameters were fitted to satisfy the dependencies of the experimental heater temperature (measured via the heater resistance) versus el ectrical current through the heater for two types of sensors (with thin about $40 \mathrm{~nm}$ membranes and thick about $400 \mathrm{~nm}$ membranes).

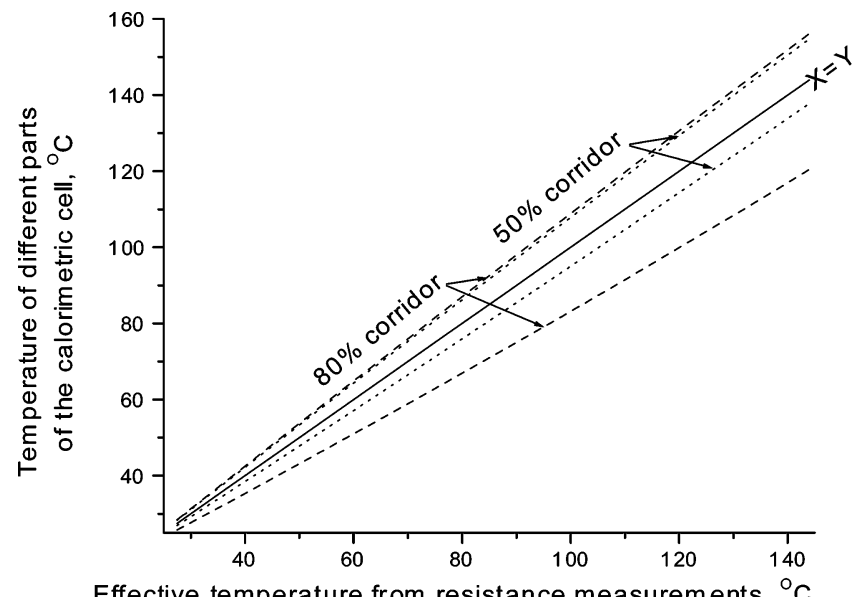

Effective temperature from resistance measurements, ${ }^{\circ} \mathrm{C}$

Figure 4. Temperature distribution over the cal orimetric cell as a function of experimentally measured temperature of the heater.

The system of the equations is solved using an iterative procedure as follows. First, the electrostatic equation is solved and the distribution of the J oule heat sources over the metallization is found. Taking into account the J oule heat source distribution and the emissive heat loss, calculated on the basis of the temperature distribution from the previous cycle, the heat balance equation is solved. According to the new temperature distribution, the conductivity of the metallization is refined and the cycle is repeated. The solution procedure is complete when the results converge (typically after a few iterations).

The calculated distribution of the temperature over the calorimetric cell as a function of experimentally measured temperature of the heater (and sample) is illustrated in Figure 4. The nonuniformity of the temperature distribution becomes more pronounced with increasing temperature, but even at $140{ }^{\circ} \mathrm{C}$ (the maximal $T_{\text {ANN }}$ for both PS and PVP) the experimental temperature gives practically nonshifted estimation of the median of the temperature distribution and more than $50 \%$ of the sample have the temperature within the range of $\pm 20 \mathrm{~K}$ of the experimental temperature.

\section{Results and Discussion}

In each annealing measurement (see Figure 2) the calorimetric curve from the first scan after annealing (1stS curve) is, in general, different from the subsequent nine scans (2ndS curves). The difference between the 1stS curve and all 2ndS curves reflects the different thermal histories of the sample. Each cal orimetric scan heats the sample well above the glass transition, and such heating "erases" the thermal history of the sample. The 1stS curve reflects a thermal history that includes both passive cooling prior to annealing and the annealing procedure itself (Figure $5 b$ ). The 2ndS curves from scans, which immediately follow the passive cooling from a previous scan, reflect a thermal history that includes only passive cooling from the glass-transition regi on to room temperature (Figure $5 \mathrm{a}$ ). Consequently, all scans which do not follow the annealing procedure (as in Figure 5a) give the same 2ndS curve throughout the whole experiment, regardless of annealing proce dures and their conditions. In contrast, 1stS curves depend on $\mathrm{T}_{\mathrm{ANN}}$. If $\mathrm{T}_{\mathrm{ANN}} \ll \mathrm{T}_{\mathrm{g}}$ (as in Figure $5 \mathrm{~b}$ ), the 1stS calorimetric curve will be cl ose to the 2ndS ones (type I of the 1stS curve). If $\mathrm{T}_{\mathrm{ANN}}>\mathrm{T}_{\mathrm{g}}$ (as in Figure $5 \mathrm{c}$ ), the previous thermal history will be erased and the scan after annealing will probe the sample with a history of cooling from the glass-transition region down to the room temperature with the rate of $2 \mathrm{~K} / \mathrm{s}$ (type II of the 

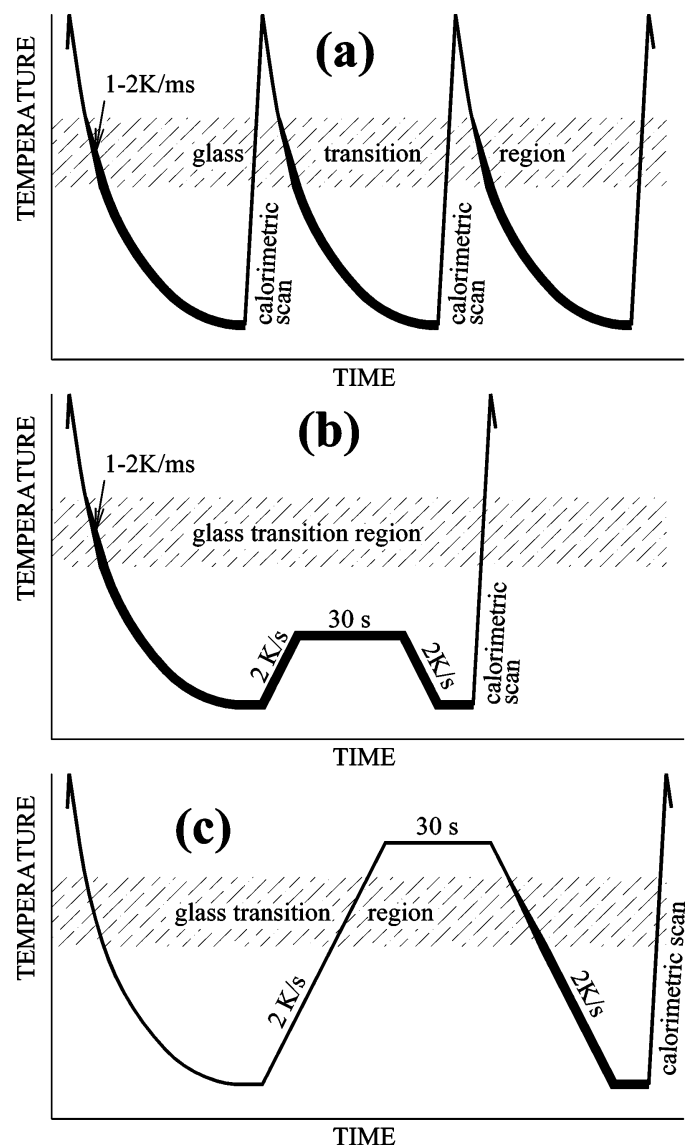

Figure 5. Different thermal histories of the sample for the cal orimetric probe. Thick part of the curve denotes the region where the thermal history of the sample forms.

1stS curve). When $\mathrm{T}_{\text {ANN }}$ approaches $\mathrm{T}_{\mathrm{g}}$, the 1stS curve will have features of both types I and II. Summarizing, the temperature of transition between type I and type II 1stS curves with change of $\mathrm{T}_{\text {ANN }}$ can be used for the assignment of $\mathrm{T}_{g}$ of the sample. Moreover, the $\mathrm{T}_{g}$ assigned in this way will reflect the properties of the sample on the time scale of the annealing procedure, not the time scale of the calorimetric scan. In this case, nanocal orimetry is used only as an indicator of changes in sample properties influenced by $T_{\text {ANN }}$, not as a direct tool to assign $T_{g}$ as a temperature of a feature on a $C_{p}(T)$ curve.

A typical series of the calorimetric curves is shown in Figure 6. As noted in section 2.2, in the case of annealing measurements, the thermal properties of the sample are presented as $\mathrm{d} \Delta \mathrm{V} / \mathrm{dt}(\mathrm{T})$ functions rather than $C_{P}(T)$ dependencies. For our analysis, the use of $\mathrm{d} \Delta \mathrm{V} / \mathrm{dt}(\mathrm{T})$ dependency is equivalent to the use of $C_{P}(T)$, and we will use the term "calorimetric curve" for $\mathrm{d} \Delta \mathrm{V} / \mathrm{dt}(\mathrm{T})$ function also. A series made using both monotonic increasing and decreasing of $\mathrm{T}_{\text {ANN }}$ are essentially the same, and repetition of these series gives the same result.

To assign a value of $T_{g}$, we need to quantify the changes of the cal orimetric curves when $T_{\text {ANN }}$ changes: $\mathrm{T}_{\mathrm{g}}$ in this approach would correspond to the change of the calorimetric curve as a whole when $\mathrm{T}_{\text {ANN }}$ crosses the glass-transition region. The parameter characterizing the curves should be a scalar that is sensitive to changes in the glass transition during the previous annealing procedure and is not sensitive to random deviations or noise in the signal. We found three parameters which satisfy these requirements.

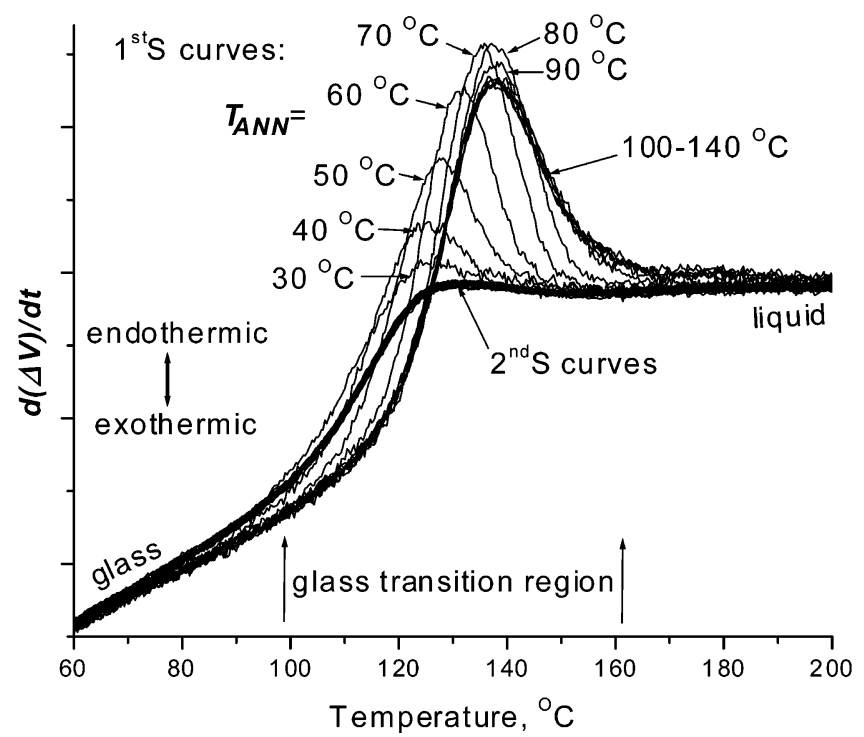

Figure 6. Series of calorimetric 1stS and 2ndS curves for $\mathrm{T}_{\text {ANN }}$ changing monotonically from $140{ }^{\circ} \mathrm{C}$ down to $30{ }^{\circ} \mathrm{C}$. Sample: $3.5 \mathrm{~nm}$ thick PS film. Curves are smoothed for clarity by 51-point second-order polynomial Savitzky-Golay filter. Curves for selected $\mathrm{T}_{\mathrm{ANN}}$ are shown only.

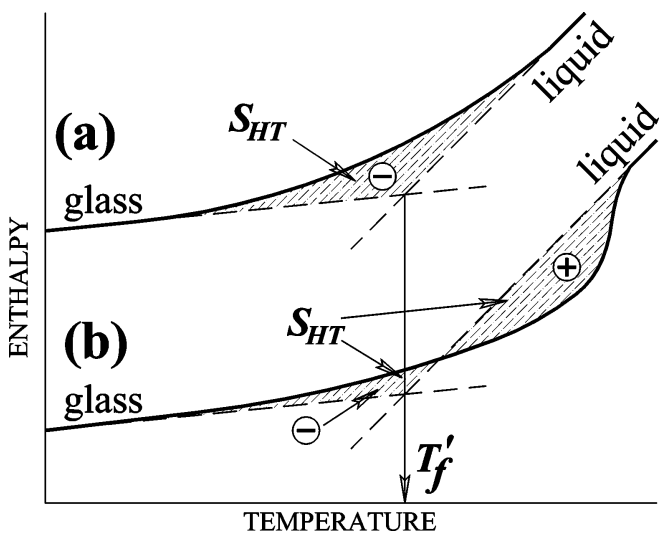

Figure 7. Limiting fictive temperature $T_{f}$ ' and "hysteresis integral" $\mathrm{S}_{\mathrm{HT}}$ definition. Two cases are illustrated: (a) "classic" glass transition with the step on $C_{P}(T)$ curve and (b) glass transition with the hysteresis peak on $C_{P}(T)$ curve. " + " and "-" denote signs of terms which form $\mathrm{S}_{H T}$.

One of these parameters is the limiting fictive temperature $T_{f}^{\prime}$ of the glass transition seen in a 1stS calorimetric curve. $T_{f}^{\prime}$ is defined as the temperature of the intersection of the extrapolated equilibrium liquid and glass $\Delta \mathrm{H}(\mathrm{T})$ curves. ${ }^{12}$ This construction is illustrated in Figure 7. $\mathrm{T}_{f}^{\prime}$ can be calculated by the following equation

$$
\int_{T_{M I N}}^{T_{f^{\prime}}}\left(C_{P}-C_{P G}\right) d T+\int_{T_{f}^{\prime}}^{T_{M A X}}\left(C_{P}-C_{P L}\right) d T=0
$$

where $T_{\text {MIN }}$ is any temperature below the glass-transition region and $T_{M A X}$ is any temperature above it, $C_{P G}$ is the glassy-state heat capacity, and $C_{P L}$ is the liquidstate heat capacity, both extrapolated to $\mathrm{T}_{\mathrm{f}}^{\prime}$. In our calculations we use $\mathrm{d} \Delta \mathrm{V} / \mathrm{dt}(\mathrm{T})$ instead of heat capacity.

Another parameter, the "hysteresis integral" $\mathrm{S}_{\mathrm{HT}}$, can be found using the following equation

$$
S_{H T}=\int_{T_{M I N}}^{T_{f}^{\prime}}\left(C_{P}-C_{P G}\right) T d T+\int_{T_{f}^{\prime}}^{T_{M A X}}\left(C_{P}-C_{P L}\right) T d T
$$




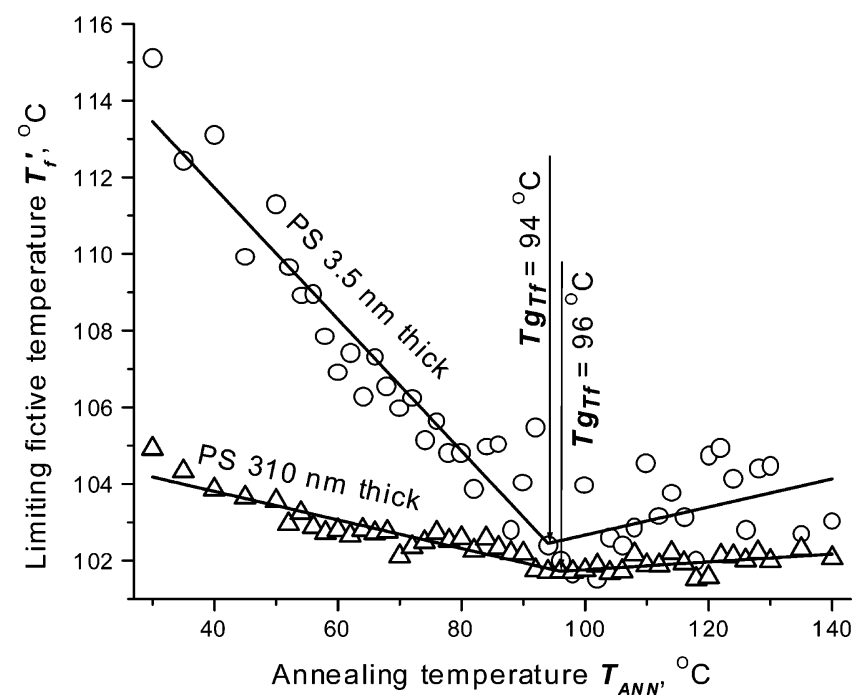

Figure 8. Dependence of $T_{f}^{\prime}$ for $1 s t S$ curves on $T_{\text {ANN }}$ : $(O)$ data for $3.5 \mathrm{~nm}$ thick PS film, $(\triangle)$ for $310 \mathrm{~nm}$ thick PS film. Straight lines denote linear fit.

This equation is invariant relative to shifts of temperature scale, so $\mathrm{T}$ can be either absolute or relative (for example, ${ }^{\circ} \mathrm{C}$ ) temperature. According to eq $2, \mathrm{~S}_{\mathrm{HT}}$ has the geometrical meaning of the signed area between the experimental $\Delta H(T)$ curve from the extrapolated equilibrium liquid and glass $\Delta H(T)$ curves. It is illustrated in Figure 7. As with $T_{f}^{\prime}$, the $d \Delta V / d t(T)$ function is used for $\mathrm{S}_{\mathrm{HT}}$ calculation instead of heat capacity.

The last parameter $\mathrm{T}_{\mathrm{PEAK}}$ is the temperature of the peak on a 1stS calorimetric curve.

Typical dependencies of $\mathrm{T}_{\mathrm{f}}^{\prime}$, calculated from 1stS curves, on $\mathrm{T}_{\text {ANN }}$ are shown in Figure 8. For $\mathrm{T}_{\text {ANN }}$ bel ow the glass-transition region, $\mathrm{T}_{\mathrm{f}}{ }^{\prime}$ decreases with increasing $\mathrm{T}_{\text {ANN. }}$. It is well known that $\mathrm{T}_{\mathrm{g}}$ decreases with an increase in the extent of the annealing treatment. ${ }^{13}$ When $T_{\text {ANN }}$ exceeds $T_{g}$, $T_{f}$ ' stabilizes-sample "forgets" the thermal history created before being cooled through the glass-transition region. This change of $T_{f}^{\prime}\left(T_{A N N}\right)$ behavior when $T_{\text {ANN }}$ crosses the glass-transition region can be used to assign $\mathrm{T}_{\mathrm{g}}$. We will call $\mathrm{T}_{\mathrm{g}}$ assigned in this way $T_{g T}$. Numerically, $T_{f}^{\prime}\left(T_{\text {ANN }}\right)$ is fitted by two intersected lines in the least-squares sense. The position of intersection gives us the $\mathrm{T}_{\mathrm{gT}_{\mathrm{f}}}$ value. This method is illustrated in Figure 8 . We reiterate that the $\mathrm{T}_{\mathrm{gT}_{\mathrm{f}}}$ value reflects the glass transition over the time scale of the annealing procedure (seconds), in contrast to the shown $T_{f}^{\prime}$ values, which reflect the time scale of TDSC scans (milliseconds).

The dependence of $\mathrm{T}_{\mathrm{g} \mathrm{T}_{\mathrm{f}}}$ on sample thickness for all investigated polymers is shown in Figure 9. The $\mathrm{T}_{\mathrm{gT}_{f}}$ values for all polymers are significantly lower than $T_{f}^{\prime}$, due to the difference in the time scales. Values of $T_{f}^{\prime}$ calculated from regular nanocal orimetric measurements using eight different heating rates in the $20-200 \mathrm{~K} / \mathrm{ms}$ heating range are shown elsewhere, ${ }^{6}$ and the average values can be found in Table 1. Here, for illustration, the $T_{f}{ }^{\prime}$ values calculated from 1stS curves with $T_{\text {ANN }}=$ $30^{\circ} \mathrm{C}$ are shown in Figure 10 . The accuracy of $\mathrm{T}_{f}^{\prime}$ shown in Figure 10 is significantly less than that demonstrated in ref 6 , due to smaller statistics, the use of only one heating rate, and negl ecting the extensive data corrections used in the accurate methods. However, the data in Figure 10, similar to the data in ref 6, show the same magnitude of $T_{f}^{\prime}$ and no significant $T_{g}$-thickness dependence for the given thickness range. (The jump in

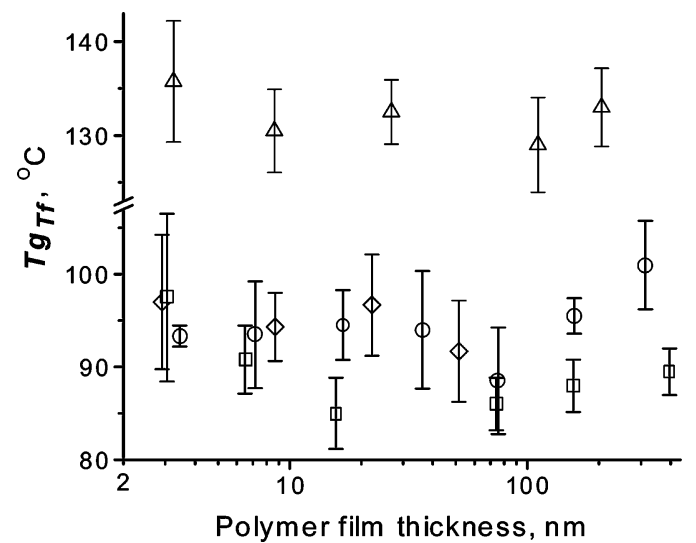

Figure 9. $T_{g}$ assigned using 1stS curves $T_{f}^{\prime}$ data $\left(T_{g T}\right)$ as a function of polymer film thickness: $(O)$ PS, $(\diamond) P S-H$, ( $\square$ ) PVP, $(\triangle)$ PMMA. Error bars represent the standard deviation of four measurements (six for PS-H).

Table 1. $T_{\mathrm{g}}$ Assigned by Different Methods

\begin{tabular}{|c|c|c|c|c|}
\hline \multirow[b]{2}{*}{ method of $\mathrm{T}_{\mathrm{g}}$ assignment } & \multicolumn{4}{|c|}{$\mathrm{T}_{\mathrm{g}}\left(\right.$ in $\left.^{\circ} \mathrm{C}\right)$ for polymers } \\
\hline & PS & PS-H & PVP & PMMA \\
\hline $\begin{array}{l}T_{f^{\prime}} \text { from regular nanocal orimetry } \\
\text { measurements using eight } \\
\text { different heating rates }{ }^{\mathrm{a} 6}\end{array}$ & 114 & 112 & 111 & 145 \\
\hline $\begin{array}{l}\mathrm{T}_{\mathrm{f}}^{\prime} \text { from anneal ing measurements, } \\
\mathrm{T}_{\text {ANN }}=30^{\circ} \mathrm{C} \text {, estimate values, } \\
\text { this paper }\end{array}$ & 110 & 115 & 104 & 136 \\
\hline $\mathrm{T}_{\mathrm{gT}_{\mathrm{f}}}$, this papera & 94 & 96 & 90 & 132 \\
\hline $\mathrm{T}_{\mathrm{gSHT}}$, this papera & 93 & 95 & 90 & 134 \\
\hline $\mathrm{T}_{\mathrm{qT}}$, this papera & 91 & 90 & 84 & 120 \\
\hline $\begin{array}{l}T_{f}^{\prime} \text { for bulk samples, conventional } \\
\text { DSC }^{14}\end{array}$ & 99 & 102 & 94 & 128 \\
\hline
\end{tabular}

a Averages for all investigated film samples starting from $3 \mathrm{~nm}$ thickness and thicker.

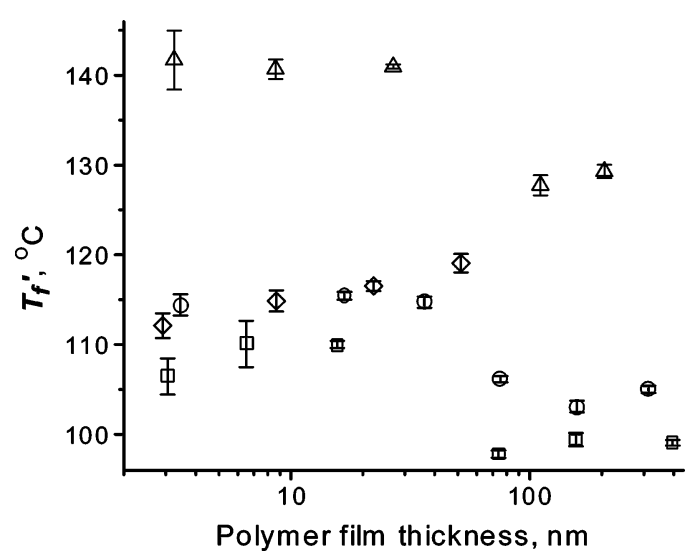

Figure 10. $\mathrm{T}_{f}^{\prime}$ calculated from 1stS curves for $\mathrm{T}_{\text {ANN }}=30^{\circ} \mathrm{C}$ as a function of polymer film thickness: $(O) \mathrm{PS} ;(\diamond) \mathrm{PS}-\mathrm{H}$, $(\square)$ PVP, $(\triangle)$ PMMA. Error bars represent the standard deviation of four measurements (six for PS-H).

$\mathrm{T}_{f}^{\prime}$, which appears around a thickness of $70 \mathrm{~nm}$, corresponds apparently to sensors with thicker membranes, used for thick films (see section 2.1). However, the reason of this jump is not clear.) On the other hand, $\mathrm{T}_{\mathrm{gT}_{\mathrm{f}}}$ are close to the bulk values determined by commercial DSC ${ }^{14}$-see Table 1. No appreciable dependence of $\mathrm{T}_{\mathrm{gT}_{\mathrm{f}}}$ on film thickness from a few nanometers up to several hundred nanometers can be seen in Figure 9. The same phenomenon-no $\mathrm{T}_{\mathrm{g}}$ thickness dependencewas observed for millisecond time scale $T_{f}^{\prime}$ data. ${ }^{6}$ It allows us to suppose that the absence of a $T_{g}$ thickness dependence is not characteristic of high heating and 


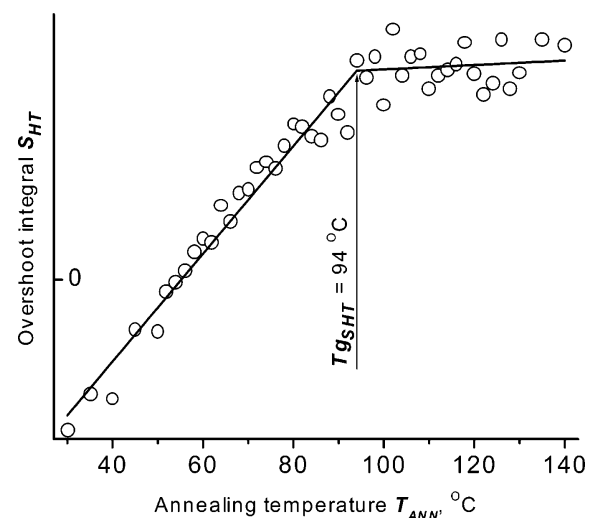

Figure 11. Dependence of hysteresis integral $\mathrm{S}_{\mathrm{HT}}$ for 1stS curves on $\mathrm{T}_{\text {ANN }}$. Sample: $3.5 \mathrm{~nm}$ thick PS film. Straight lines denote linear fit.

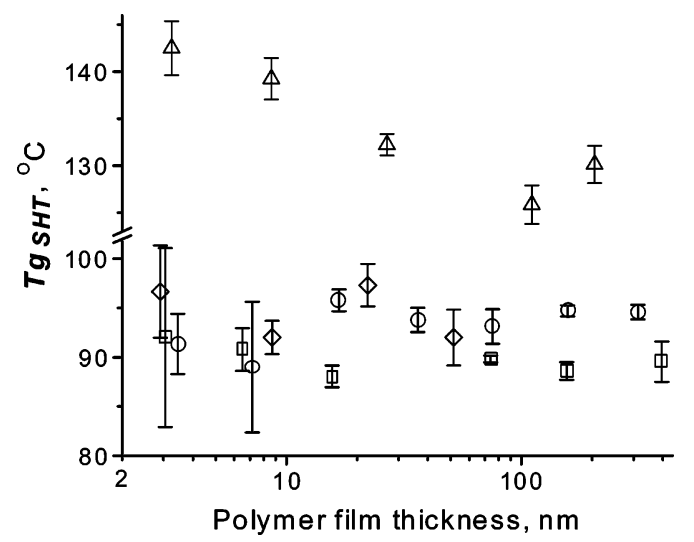

Figure 12. $T_{g}$ assigned using 1 stS curves $S_{H T}$ data $\left(T_{g S_{H T}}\right)$ as a function of polymer film thickness: (O) PS; $(\diamond) \mathrm{PS}-\mathrm{H}$, ( $\square$ ) PVP,$(\triangle)$ PMMA. E rror bars represent the standard deviation of four measurements (six for $\mathrm{PS}-\mathrm{H}$ ).

cooling rates (or small time scale) but is a more general phenomenon.

There is the same situation for hysteresis integral $\mathrm{S}_{\mathrm{HT}}$. A typical dependence of $\mathrm{S}_{\mathrm{HT}}$, calculated from 1stS curves, on $\mathrm{T}_{\text {ANN }}$ is shown in Figure 11 . For $\mathrm{T}_{\text {AnN }}$ below the glass-transition region, the overshooting (hysteresis peak, visible in Figure 6) on a calorimetric curve obtained from a DSC scan upon heating increases with increasing difference between cooling and subsequent heating rates or, equivalently, with increasing of the extent of the annealing treatment while the heating conditions remain the same. ${ }^{12,13}$ When $T_{\text {ANN }}$ exceeds $T_{g}$, the calorimetric 1stS curve (and the hysteresis peak on it) stabilizes. As in the $T_{f}^{\prime}\left(T_{\text {ANN }}\right)$ case, this feature on a $\mathrm{S}_{\mathrm{HT}}\left(\mathrm{T}_{\mathrm{ANN}}\right)$ function can be used to assign $\mathrm{T}_{g}$, using the same method we described for $\mathrm{T}_{\mathrm{gT}_{\mathrm{f}}}$ (see Figure 11). We will denote $T_{g}$ assigned using $\mathrm{S}_{\mathrm{HT}}\left(\mathrm{T}_{\mathrm{ANN}}\right)$ as $\mathrm{T}_{\mathrm{gS} \mathrm{HT}}$.

The dependence of $\mathrm{T}_{\mathrm{gS}}$ on sample thickness for all investigated polymers is shown in Figure 12. The $\mathrm{T}_{\mathrm{gS}} \mathrm{HT}$ values are close to $\mathrm{T}_{\mathrm{gT}}$, shown in Figure 9 , and also have no appreciable dependence on film thickness. $T_{g T_{f}}$ for low thickness PMMA films slightly deviates both from $\mathrm{T}_{\mathrm{gS}}$ for thicker films and from $\mathrm{T}_{\mathrm{gT}_{\mathrm{f}}}$ data. Supposedly this deviation is caused by increased error of $\mathrm{T}_{\mathrm{gS}} \mathrm{S}_{\mathrm{HT}}$ evaluation for films with thickness bel ow $10 \mathrm{~nm}$, as the increased error bars for PS and PVP films suggest.

The $T_{g S_{H T}}$ data also support the conclusion made for $\mathrm{T}_{\mathrm{gT}}$ that there is no appreciable thickness dependence of $\mathrm{T}_{\mathrm{g}}$ for large (seconds) time scale DSC measurements.

The same discussion can be repeated for the TPEAK parameter. A typical dependence of TPEAK, calculated

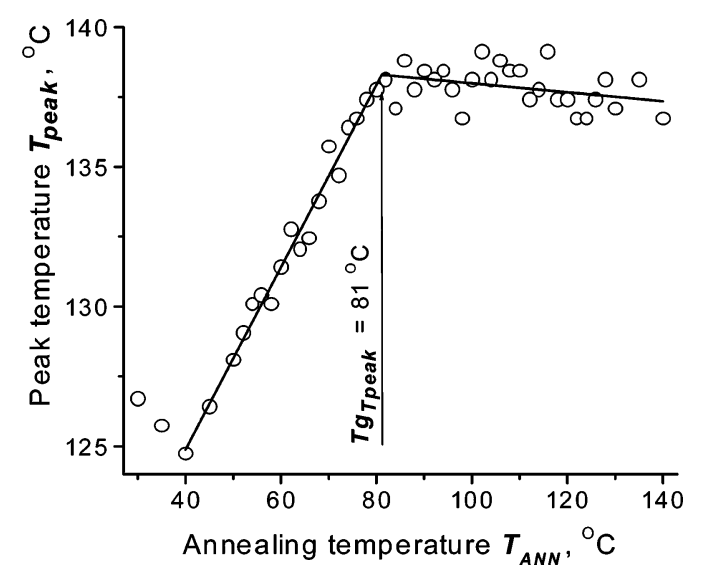

Figure 13. Dependence of the peak temperature $T_{P E A K}$ for 1stS curves on $T_{A N N}$. Sample: $3.5 \mathrm{~nm}$ thick PS film, first run down. Straight lines denote linear fit.

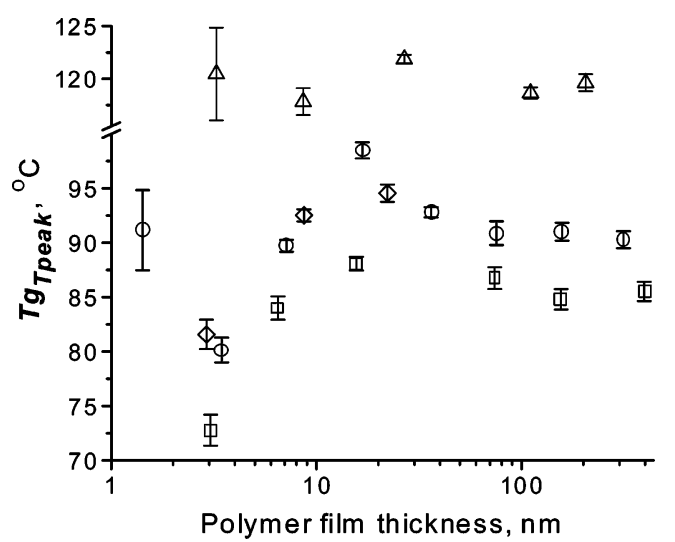

Figure 14. $T_{g}$ assigned using 1 stS curves $T_{P E A K}$ data $\left(T_{g T_{P E A K}}\right)$ as a function of polymer film thickness. (O) PS; $(\diamond) P S-H,(\square)$ PVP, $(\triangle)$ PMMA. Error bars represent the standard deviation of four measurements (six for PS-H).

from 1stS curves, on $T_{\text {ANN }}$ is shown in Figure 13. As mentioned for $\mathrm{S}_{\mathrm{HT}}$, the hysteresis peak on a calorimetric curve increases with the extent of the annealing treatment. While $T_{f}^{\prime}$ is less susceptible to changes in thermal history, the increasing hysteresis peak should shift to higher temperatures in order to keep $\mathrm{T}_{f}^{\prime}$ relatively constant. When $T_{\text {ANN }}$ exceeds $T_{g}, T_{\text {PEAK }}$ stabilizes. The same method, as for $\mathrm{T}_{f}^{\prime}\left(\mathrm{T}_{\mathrm{ANN}}\right)$ and $\mathrm{S}_{\mathrm{HT}}\left(\mathrm{T}_{\mathrm{ANN}}\right)$, can be used to assign $T_{g}$, as can be seen in Figure 13. The result will be denoted as $\mathrm{T}_{\text {gT PEAK }}$.

The dependence of T TT PEAK on sample thickness for all investigated polymers is shown in Figure 14. $T_{\text {gT }} T_{\text {PEAK }}$ for PMMA is about $10 \mathrm{~K}$ less than $T_{g T_{f}}$ and $T_{g S_{H T}}$ values. There is no significant dependence of $T_{\text {gT }} T_{\text {PEAK }}$ on film thickness, as in other two cases, but there is noticeable drop (about $10 \mathrm{~K}$ ) in $\mathrm{T}_{\mathrm{gT}}$ PEAK for PS, PS-H, and PVP at a film thickness of about $3 \mathrm{~nm}$. It should be taken into consideration that while the first two parameters discussed above $\left(T_{f}^{\prime}\right.$ and $S_{H T}$ ) are integral ones and incorporate information from the significant part of cal orimetric curve, $T_{\text {PEAK }}$ characterizes only a small part of the curve-the top of the glass-transition hysteresis peak - and, therefore, is less accurate. It also can be seen in Table 1 that average val ues of $\mathrm{T}_{\mathrm{gT}}$ and $\mathrm{T}_{\mathrm{gS}} \mathrm{HT}$ are close to each other, but there is a noticeable difference between $\mathrm{T}_{\text {gT }} \mathrm{T}_{\text {PEAK }}$ and both $\mathrm{T}_{\mathrm{gT}}$ and $\mathrm{T}_{\mathrm{gS}}$; ; additionally, both $\mathrm{T}_{\mathrm{gT}}$ and $\mathrm{T}_{\mathrm{g} \mathrm{S}_{\mathrm{H}}}$ values are closer to the bulk $\mathrm{T}_{\mathrm{g}}$ than $T_{\text {gT PEAK }}$ is.

ThuS, for $T_{g T_{f}}, T_{g S H T}$, and $T_{g T_{P E A K}}$, no appreciable dependence on film thickness is found for these mea- 
surements on the time scale of seconds. Small, on the order of $10 \mathrm{~K}$, deviations in $\mathrm{T}_{\mathrm{gT}} \mathrm{T}_{\mathrm{PEA}}$ for some polymers may be attributed to decreased reliability of $\mathrm{T}_{\mathrm{g}}$ assignment using TPEAK data.

Returning to the initial problem addressed by this work, it should be noted that one of the main results of our previous work ${ }^{6}$ is the absence of a dependence of $T_{g}$ on the film thickness down to a few nanometers. This conclusion is the result of the investigation of supported PS, PS-H, PVP, and PMMA films on a platinum surface by the nanocal orimetric technique. On the other hand, ellipsometric measurements (which became de facto the standard method for investigation of $\mathrm{T}_{\mathrm{g}}$ thickness dependence in supported polymer films), dielectric measurements, and several other methods show significant dependence of $T_{g}$ on film thickness. One of the ideas devoted to explain this discrepancy considers the difference in the time scale of the glass transition investigated by nanocalorimetry and by ellipsometry. High heating rates, intrinsic to the TDSC method, make the time scale of the observed glass transitions on the order of milliseconds. Indeed, assuming a temperature interval of a glass transition of about $40 \mathrm{~K}$ and using a typical TDSC heating rate of $20-200 \mathrm{~K} / \mathrm{ms}$, the time of crossing the glass-transition region is $0.2-2 \mathrm{~ms}$. A typical rate of temperature change in ellipsometric measurements is $0.2-2 \mathrm{~K} / \mathrm{min}^{15-17}$ and in dielectric measurements is $0.5-3 \mathrm{~K} / \mathrm{min} .{ }^{15,18,19}$ These rates correspond with a glass-transition time scale of minutes. Hartmann and coauthors ${ }^{20}$ show explicitly on the example of spin-cast i-PMMA films that, for the thickness range from the bulk down to $20 \mathrm{~nm}$, the dependence of the maximum $\tau_{\max }$ of the relaxation time distribution on the film thickness (activation plots) is negligible for $\tau_{\max } \approx 0.1-1 \mathrm{~ms}$. Thickness dependence of $\tau_{\max }$ became pronounced when $\tau_{\max }$ approaches the magnitude of seconds and more. Clearly, these results lead to the supposition that a glass-transition temperature on a milliseconds ti me scale would have negligible thickness dependence and became more pronounced for time scales of seconds and minutes.

Apparently, the results presented here do not support this hypothesis. The time scale of the glass-transition phenomenon in the annealing experiments can be estimated from the heating/cooling rate in the annealing treatment, $2 \mathrm{~K} / \mathrm{s}$. Using the same glass-transition interval as we used above, $40 \mathrm{~K}$, the time scale of the glass-transition process in the presented experiments is about $20 \mathrm{~s}$, which is more than four orders of magnitude greater than the time scale in the previous nanocal orimetric measurements. The absence of the $T_{g}$ thickness dependence in both cases is an argument against the supposition mentioned above.

Literature data on the cal orimetry of the small-sized samples of polymers used in this work are very limited but consistent with our results. A recent calorimetry study of small PS spheres in aqueous suspensions, ${ }^{21}$ where the authors overcome spurious coalescence effects, shows no substantial depression of $T_{g}$ of PS spheres for the investigated range of diameters 42-548 $\mathrm{nm}$. Adsorbed PS on silica with an equivalent average thickness estimated as $0.8-2 \mathrm{~nm}$, investigated by a modulated DSC technique, demonstrates only a slight rise of $T_{g}, 4-6 \mathrm{~K}$ above the bulk $\mathrm{T}_{\mathrm{g}}{ }^{22}$ Interestingly, this increase in $\mathrm{T}_{\mathrm{g}}$ probably corresponds with the increased $(10-15 \mathrm{~K}) \mathrm{T}_{\mathrm{g}}$ for the smallest $(1-1.5 \mathrm{~nm})$ samples in our nanocal orimetry work. ${ }^{6}$
A reasonable explanation of this seeming contradiction-that some experimental methods show a significant $T_{g}$ depression in thin polymer films while other ones, including calorimetry, do not-has appeared in recent publications by F orrest, ${ }^{1}$ Porter and Blum, ${ }^{22}$ and Kawana and $\mathrm{J}$ ones ${ }^{17}$ and can be formulated as follows. While the temperature of a first-order thermodynamic transition (e.g., the melting of a crystal) can be measured, the glass-transition temperature $T_{g}$, due to the dynamic nature of glass transition, can only be assigned. The assignment of $\mathrm{T}_{\mathrm{g}}$ using the concept of limiting fictive temperature $T_{f}^{\prime}$, which was described above, depends only on the equilibrium liquid and glass enthal py-temperature dependencies, so it is not sensitive to complex relaxation phenomena in the vicinity of the glass transition and is widely used both in experimental and theoretical works. ${ }^{12,13,23}$ Both our previous work with nanocal orimetry ${ }^{6}$ and the calorimetry of small PS spheres ${ }^{21}$ use this assignment to observe $\mathrm{T}_{\mathrm{g}}$-thickness dependence; the calorimetry of adsorbed PS uses a $T_{g}$ definition which should yield values very close to $T_{f}{ }^{\prime}$ for the presented calorimetric curves.

Other methods typically use arbitrary features of the experimental curves to assign $\mathrm{T}_{\mathrm{g}}$. We can expect that certain $\mathrm{T}_{\mathrm{g}}$ assignments are more sensitive to the onset of the glass transition. ${ }^{22}$ These procedures yield values of $\mathrm{T}_{\mathrm{g}}$ onset, which can be close to other assignments and to $T_{f}^{\prime}$, if the breadth of glass transition is small. Numerous investigations ${ }^{1-4}$ including the previously mentioned calorimetry works on nanoscale PS objects ${ }^{6,22}$ demonstrate pronounced broadening of glass transition with decreasing film thickness. The broadening effect obviously causes a low-temperature shift of the beginning of the glass transition and, consequently, the $T_{g}$ onset depression, even if $\mathrm{T}_{f}^{\prime}$ is constant. For the ellipsometry technique and PS spin-coated films it is shown that $T_{g}$ assigned as the onset temperature of the expansivitytemperature function demonstrates significant depression with decreasing thickness while the endpoint temperature of the glass-transition, $\mathrm{T}_{\mathrm{g}}$ endpoint, remains constant or slightly increases. ${ }^{17}$

Interestingly, a simple computational experiment demonstrates the same trends for $\mathrm{T}_{\mathrm{g}}{ }^{\text {onset }}$ and $\mathrm{T}_{\mathrm{g}}$ endpoint. Using the standard Tool-Narayanaswamy-Moynihan (TNM) model, ${ }^{5}$ we calculate $T_{f}(T)$ function for virtual samples: bulk PS and PS films with different thicknesses $\mathrm{d}$. Thermal history starts at $\mathrm{T}_{\text {MAX }}=240^{\circ} \mathrm{C}$ (when the samples are liquid); the virtual samples are cooled to $\mathrm{T}_{\text {MIN }}=20^{\circ} \mathrm{C}$ (when the samples are glassy) with the rate $\mathrm{v}_{\text {cooling }}=-2 \mathrm{~K} / \mathrm{s}$ and then heated to $\mathrm{T}_{\text {MAX }}$ with the rate $v_{\text {heating }}=2 \mathrm{~K} / \mathrm{s}$. Parameters for bulk PS modeling are taken from ref 23 Table 1, using average values for the polymer: activation enthalpy $\Delta h * / R=76 \mathrm{kK}$, stretch exponent $\beta_{\infty}=0.69$, nonlinearity parameter $\mathrm{x}=0.48$, and limiting fictive temperature $\mathrm{T}_{f_{\infty}^{\prime}}=$ $100{ }^{\circ} \mathrm{C}$. This set of values defines the fourth TNM parameter, the exponent coefficient $A_{E X P}\left(\log A_{E X P}=\right.$ $-87.53, A_{\text {EXP }}$ given in seconds). We assume that film samples have bulk values of $\Delta h * / R, x$, and $A_{E X P}$. The broadening of the glass transition in film samples with decreasing of $d$ is introduced as a thickness dependence of the stretch exponent value, which characterizes the broadness of the relaxation time spectrum corresponding with glass transition: $\beta(\mathrm{d})=\beta_{\infty} \exp (-\mathrm{a} / \mathrm{d})$, where $a=4.9 \mathrm{~nm} .{ }^{6}$ Limiting the fictive temperature for the film samples, $\mathrm{T}_{\mathrm{f}}(\mathrm{d})$ changes with $\beta(\mathrm{d})$. F or $\mathrm{T}_{\mathrm{g}}$ assignment, we use the heating part of the thermal history 


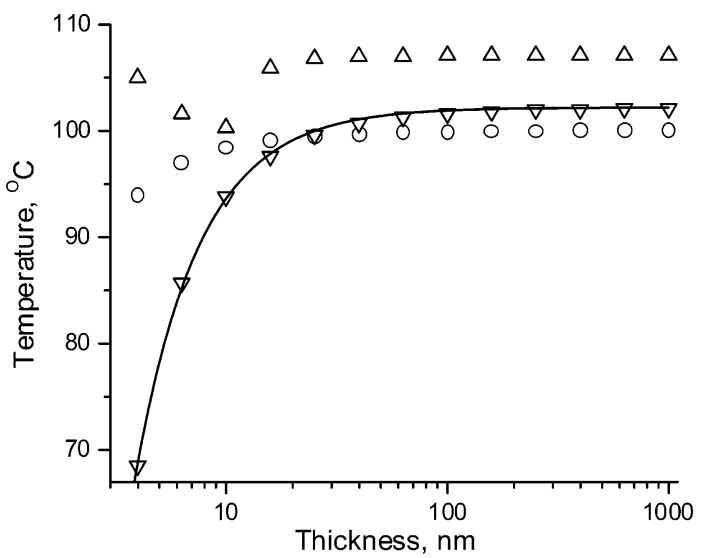

Figure 15. Output of the computational experiment with virtual PS films: $T_{g}$ assigned by different methods as functions of film thickness d: (O) $\mathrm{T}_{f}^{\prime}(\mathrm{d}),(\Delta) \mathrm{T}_{\mathrm{g}}$ endpoint, $(\nabla) \mathrm{T}_{\mathrm{g}}$ onset. Solid line denotes the best fit for $T_{g}$ onset(d) dependence using the equation $\mathrm{T}_{\mathrm{g}}$ onset $(\mathrm{d})=\mathrm{T}_{\mathrm{g}}{ }^{\text {onset }}\left(\left(1-(\mathrm{A} / \mathrm{d})^{\delta}\right)\right.$. The best-fit parameters are $\mathrm{A}=0.8 \mathrm{~nm}, \delta=1.5$.

and two straightforward criteria. We assign $\mathrm{T}_{\mathrm{g}}$ onset as the upper limit of the interval $\left[T_{M I N}, T\right]$ where the fictive temperature $T_{f}$ is close to its glass value $T_{f}$ : $\left|T_{f}-T_{f}\right|<1 \mathrm{~K}$, and $T_{g}$ endpoint as the lower limit of the interval $\left[T, T_{\text {MAX }}\right.$ ] where $T_{f}$ approaches the real temperature $T$ (this is characteristic for the liquid state): $\left|T_{f}-T\right|<1 \mathrm{~K}$. The result is shown in Figure 15. Even for this simplistic model and rather arbitrary criteria for $\mathrm{T}_{\mathrm{g}}$ onset and $\mathrm{T}_{\mathrm{g}}$ endpoint, we can see the same trends as discussed above: while $T_{f}{ }^{\prime}$ is slightly decreasing with a decrease in $d, T_{g}$ endpoint remains almost constant and $\mathrm{T}_{\mathrm{g}}$ onset falls significantly. Note that the $\mathrm{T}_{\mathrm{g}}$ onset trend is well-fitted by the standard empirical function for $T_{g}$ depression: $T_{g}(d)=T_{\text {goo }}(1-(A / d) \delta)$, where $T_{\text {goo }}$ is $T_{g}$ bulk value, $\mathrm{A}$ and $\delta$ are fitting parameters. ${ }^{24}$ The best fit $\mathrm{A}$ and $\delta$ parameters are close to the literature values: compare $\mathrm{A}=0.8 \mathrm{~nm}$ from our data and $0.83 \mathrm{~nm}$ from ref $25, \delta=1.5$ from our data and literature values $1.1,17$ 1.8, ${ }^{24}$ and 2.0. ${ }^{26}$ The data obtained do not depend on the cooling/heating rate when $v_{\text {heating }}=-v_{\text {cooling; }}$; any factor will change only the scaling parameter $A_{E X P}$.

In a conclusion, recent experimental works and even simple modeling supports the hypothesis that the experimental $\mathrm{T}_{\mathrm{g}}$ trend depends on the type of $\mathrm{T}_{\mathrm{g}}$ assignment and reflects the broadening of glass transition rather than a shift of the transition region.

Some publications have experimental procedures that are described in sufficient detail to show whether the $\mathrm{T}_{\mathrm{g}}$ assignment used in a given work yields $\mathrm{T}_{\mathrm{g}}{ }^{\text {onset. }}$. One excellent example is the work ${ }^{27}$ where so-called local thermal analysis is employed to observe the glass transition in PS, PMMA, and some other polymeric spincast films. The authors ${ }^{27}$ state explicitly that $T_{g}$ is assigned using the onset on the property-temperature diagrams. Moreover, the authors make another $\mathrm{T}_{\mathrm{g}}$ assignment using the standard ellipsometry measurements and find that both methods agree and show the same $\mathrm{T}_{\mathrm{g}}$ depression. In our opinion, this is more evidence that standard ellipsometry-based $\mathrm{T}_{g}$ assignment is biased to the beginning of glass transition. It is al so shown ${ }^{27}$ that the $T_{g}$ values assigned in these ways for thick films are in agreement with bulk DSC measurements, which supports our supposition that $T_{g}$ onset for bulk samples, where the glass-transition region is relatively narrow, can be close to the results obtained by traditional methods. Another example, where $\mathrm{T}_{\mathrm{g}}$ seems to be assigned close to the onset and shows a depression trend in a good agreement with ellipsometry measurements, is the work in ref 28; the thermal stress of the PS on $\mathrm{Si}$ is measured against temperature, and corresponding diagrams show details of the $\mathrm{T}_{\mathrm{g}}$ assignment method.

In contrast, the method of $\mathrm{T}_{\mathrm{g}}$ assignment used in the present work relies instead on the endpoint of the glass transition, where liquefying of the sample erases its thermal history. A similar approach is used in ref 7: poly(vinyl alcohol) samples with a mechanically treated surface are annealed and sum-frequency vibrational spectroscopy (SFS) is used to test whether the annealing erases the traces of the treatment. It is found that surface $T_{g}$ is essentially the same as the bulk one.

The annealing approach described here is not limited to nanocal orimetry or SFS. It would be interesting to use the annealing experiments in combination with the techniques, which show $\mathrm{T}_{\mathrm{g}}$ depression (ellipsometry, etc.). We suppose that these experiments would also yield more flat $\mathrm{T}_{\mathrm{g}}(\mathrm{d})$ dependencies.

It also should be noted that $T_{g}$ assignment for extremely thin films typically yields less reliable results in comparison with thicker films. Besides the poor signal-to-noise ratiotypical for minusculesamples, there are several other effects that complicate the $\mathrm{T}_{\mathrm{g}}$ assignment in thin films. The phenomenon of loss of glasstransition contrast in thin films has al ready been used to resolve controversies in thin-film $\mathrm{T}_{\mathrm{g}}$ studies. ${ }^{2} \mathrm{~L}$ imited temperature range of glass-transition observation and curvature of the property-temperature plots outside of glass-transition region are discussed el sewhere. ${ }^{17}$ These effects can contribute a significant part in apparent trend of $T_{g}$-thickness dependence.

Several other interesting results of presented annealing experiments should also be mentioned. All three $T_{g}$ versus thickness plots (Figures 9, 12, and 14) demonstrate no noticeable difference in $\mathrm{T}_{\mathrm{g}}$ among pol ystyrene of a different molecular weight (PS and PS-H). The same phenomenon-the absence of $\mathrm{T}_{\mathrm{g}}$ dependence on the molecular weight-was shown for the same pol ymers for cal orimetric experiments with millisecond time scal es. ${ }^{6}$ This phenomenon is commonly supported by glasstransition investigations of thin polymer films on substrates. ${ }^{1}$

It is worth noting that the annealing experiments discussed here may serve as a sensitive technique to observe glass transitions in ultrathin polymer films, where regular nanocalorimetry measurements give doubtful results. Annealing experiments we have shown here are characterized by an extremely high ratio of heating and cooling rates, about $2 \times 10^{4}$. In these conditions, the overshooting peak corresponded to the glass transition becaming significantly more pronounced than in the regular nanocal orimetric measurements, which have heating/cooling rate ratios of about 10-40 in the 100-200 ${ }^{\circ} \mathrm{C}$ temperature range. Figure 16 demonstrates that the glass transition in a $0.8 \mathrm{~nm}$ thick PVP film is hardly visible on a regular nanocal orimetric curve (2ndS curve) while the annealing 1stS curve gives clear evidence of the existence of the glass transition in this sample. Note that the given thickness of $0.8 \mathrm{~nm}$ is only an average value, measured in this case by ellipsometric measurements. A roughness of $1.5 \mathrm{~nm}$ (see section 2.3) means that the value of $0.8 \mathrm{~nm}$ cannot be treated as a local film thickness. 


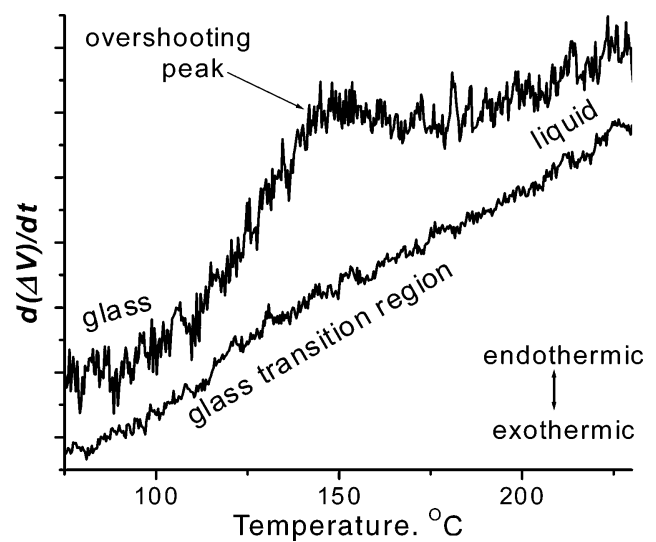

Figure 16. Calorimetric 1stS (upper) and 2ndS (lower) curves for PVP film with average thickness of about $0.8 \mathrm{~nm}$. $\mathrm{T}_{\text {ANN }}=$ $80^{\circ} \mathrm{C}$. Curves are smoothed for clarity by 31-point second-order polynomial Savitzky-Golay filter. The 2ndS curve is the average of eight scans. The heating/cool ing rate ratio is about 25 for 2ndS curve and about 20000 for 1stS curve. Curves offset for clarity.

Our attempts to fit measured calorimetric curves using the TNM model, as we did in our previous works, 5,6 were not successful. While fitting of $C_{p}(T)$ obtained by different scan rates and the same cooling history gives reasonable results, the fitting of calorimetric curves obtained using identical heating but different cooling histories (different $T_{\text {ANN }}$ ) meets serious problems. Figures $6,8,11$, and 13 show that the shape of the 1stS calorimetric curves and their characteristic parameters change starting at the lowest $\mathrm{T}_{\mathrm{ANN}}, 30^{\circ} \mathrm{C}$, and the parameters change almost linearly up to $\mathrm{T}_{\mathrm{g}}$. This behavior suggests a broad distribution of characteristic relaxation times with a smeared maximum(s). In contrast, fitting narrow and pronounced hysteresis peak upon heating gives a relatively narrow relaxation time spectrum (i.e., parameter $\beta$ in the stretched exponent form of the relaxation function is close to 1 ), especially for thick films. ${ }^{5,6}$ This may indicate the lack of thermorheological simplicity ${ }^{23}$ in the case of combining data from very different time scales in the same fit: the shape of the relaxation time distribution effectively changes when we go from the annealing procedure with a time scale of seconds to the nanocal orimetric scan with a time scale of milliseconds. We expect that broad timescale nanocalorimetry, which is under development, would address this intriguing issue.

\section{Conclusions}

The glass-transition temperature $T_{g}$ is measured in thin films of PS, PS-H, PVP, and PMMA polymers in the thickness range from hundreds of nanometers down to a few nanometers. A modification of the TDSC method is used in which the calorimetric curves are recorded as a function of the maximal temperature $T_{\text {ANN }}$ of the annealing treatment of the film tested. The $T_{g}$ is assigned as an annealing temperature where the behavior of calorimetric curves with respect to $T_{\text {ANN }}$ changes. Three parameters are used to represent changes in the curves with $\mathrm{T}_{\mathrm{ANN}}$ : limiting fictive temperature $\mathrm{T}_{f}^{\prime}$, hysteresis integral $\mathrm{S}_{\mathrm{HT}}$, and peak temperature TPEAK.

$\mathrm{T}_{\mathrm{g}}$ assigned by this method corresponds to the time scale of the glass transition, which is different from the time scale of glass transition investigated by the regular TDSC technique, used in previous work. ${ }^{6}$ The regular
TDSC technique is characterized by high heating rates (20-200 K/ms); in the calorimetric scan, the sample crosses the glass-transition region in about $1 \mathrm{~ms}$ or less. The annealing modification to the TDSC technique gives the characteristics of the glass transitions that occurred during the annealing treatment of the sample; the calorimetric curves are used only as an indicator of changes in the state of the sample caused by annealing. Consequently, $\mathrm{T}_{\mathrm{g}}$ obtained by the annealing modification of TDSC represents the time scale of the annealing treatment, which is seconds in this investigation. This time scale is cl ose to the time scale of conventional DSC calorimeters-seconds or minutes.

Regardless of more than 3 orders of magnitude difference between the time scales of the regular and modified TDSC technique, the annealing measurements give essentially the same results as regular TDSC. No appreciable dependence of $\mathrm{T}_{\mathrm{g}}$ on film thickness from hundreds of nanometers down to $3 \mathrm{~nm}$ is found for all investigated polymers. No molecular weight dependence of $T_{g}$ is observed for polystyrene films. The difference in absolute values of $\mathrm{T}_{\mathrm{g}}$ between these two methods is apparently explained by the difference in the heating/ cooling rate ratio for each of these techniques.

Additionally, the annealing modification of the TDSC exaggerates the overshooting peak, typical for glass transition under high heating/cooling rate ratio conditions. This effect can be used to observe glass transition in extremely thin films, where the regular TDSC gives indistinct results.

Acknowledgment. We gratefully acknowledge $\mathrm{P}$. Infante of CNF at Cornell University for assistance in the fabrication of the sensors and S. Granick and P. Geil from the University of Illinois at Urbana-Champaign for valuable discussions. We al so greatly benefited from hel pful discussions with J . Forrest from the U niversity of Waterloo, Canada, who attracted our attention to ref 20 and the importance of time scale considerations. This work is supported by the US NSF-DMR 0108694 and ACS-PRF \#37027-AC7. The calorimetry equipment was developed under NSF 9803019.

\section{References and Notes}

(1) Forrest, J . A. Eur. Phys. J . E 2002, 8, 261.

(2) Forrest, J . A.; Dalnoki-Veress, K. Adv. Colloid Interface Sci. 2001, 94, 167.

(3) McKenna, G. B. J . Phys. IV Fr. 2000, 10, Pr7-53 and Pr7343.

(4) Forrest, J . A.; J ones, R. A. L. I n Polymer Surfaces, I nterfaces and Thin Films; Karim, A., Kumar, S., Eds.; World Scientific: Singapore, 2000; p 251.

(5) Efremov, M. Yu.; Warren, J. T.; Olson, E. A.; Zhang, M.; Kwan, A. T.; Allen, L. H. Macromolecules 2002, 35, 1481.

(6) Efremov, M. Yu.; Olson, E. A.; Zhang, M.; Zhang, Z.; Allen, L. H. Phys. Rev. Lett. 2003, 91, 085703.

(7) Zhang, C.; Hong, S.-C.; J i, N.; Wang, Y.-P.; Wei, K.-H.; Shen, Y. R. Macromolecules 2003, 36, 3303.

(8) Olson, E. A.; Efremov, M. Yu.; Zhang, M.; Zhang, Z.; Allen, L. H. MEMS J . 2003, 12, 355.

(9) Efremov, M. Yu.; Olson, E. A.; Zhang, M.; Lai, S. L.; Schiettekatte, F.; Zhang, Z. S.; Allen, L. H. Thermochim. Acta 2004, 412, 13.

(10) Efremov, M. Yu.; Olson, E. A.; Zhang, M.; Schiettekatte, F.; Zhang, Z. S.; Allen, L. H. Rev. Sci. Instrum. 2004, 75, 179.

(11) Partial Differential Equation Toolbox User's Guide; The MathWorks, Inc.: Natick, MA, 1995.

(12) Moynihan, C. T. In Assignment of the Glass Transition, ASTM STP 1249; Seyler, R. J., Ed.; American Society for Testing and Materials: Philadel phia, 1994; p 32. 
(13) Wunderlich, B. In Assignment of the Glass Transition, ASTM STP 1249; Seyler, R. J ., Ed.; American Society for Testing and Materials: Philadel phia, 1994; p 17.

(14) $T_{f}^{\prime}$ for bulk quantity of used substances is measured using Perkin-EImer DSC7, calibrated against the melting temperature of In and Ga; polymer samples are 5-20 mg, and data are collected during heating with rate $3 \mathrm{~K} / \mathrm{min}$.

(15) Sharp, J . S.; Forrest, J . A. Phys. Rev. E 2003, 67, 031805.

(16) Herminghaus, S.; J acobs, K.; Seeman, R. Eur. Phys. J . E 2001, 5, 531

(17) Kawana, S.; J ones, R. A. L. Phys. Rev. E 2001, 63, 021501.

(18) Fukao, K.; Miyamoto, Y. Phys. Rev. E 2000, 61, 1743.

(19) Fukao, K.; Uno, S.; Miyamoto, Y.; Hoshino, A.; Miyaji, H. J. Non-Cryst. Solids 2002, 307-310, 517.

(20) Hartmann, L.; Gorbatschow, W.; Hauwede, J .; Kremer, F. Eur. Phys. J. E 2002, 8, 145

(21) Sasaki, T.; Shimizu, A.; Mourey, T. H.; Thurau, C. T.; Ediger, M. D. J . Chem. Phys. 2003, 119, 8730.
(22) Porter, C. E.; Blum, F. D. Macromolecules 2002, 35, 7448.

(23) Hodge, I. M. J . Non-Cryst. Solids 1994, 169, 211.

(24) Keddie, J . L.; J ones, R. A. L.; Cory, R. A. Europhys. Lett. 1994, 27, 59.

(25) $\mathbf{A}$ is extracted from the review, ${ }^{17}$ Figure 1 . The value presented in the caption of the figure $(8.3 \mathrm{~nm})$ is not correct. It should be decreased by a factor of 10 , as our analysis of the figure suggests.

(26) Ellison, C. J .; Torkelson, J . M. Nat. Mater. 2003, 2, 695.

(27) Fryer, D. S.; Nealey, P. F.; de Pablo, J . J . Macromolecules 2000, 33, 6439.

(28) Zhao, J.-H.; Kiene, M.; Hu, C.; Ho, P. S. Appl. Phys. Lett. 2000, 77, 2843.

MA035909R 